\title{
JOURNAL OF THE ROYAL STATISTICAL SOCIETY
}

\section{SERIES B - METHODOLOGICAL}

Vol. 37, No. 2

Techniques for testing the constancy of regression relationships over time (with Discussion) R. L. Brown, J. Durbin and J. M. Evans

Bayes equivariant estimators in high order hierarchical random effects models $H$. Sahai A sequential test for certain composite hypotheses J. Whitehead

Equivalence theorems for polynomial-projecting predictors E.J.Godolphin and P.J. Harrison

Components of Cramer - von Mises statistics. II. J. Durbin, M. Knott and C."C. Tay/or

Some new results for Poisson cluster processes $D$. Oakes

On the concepts of sufficiency and ancillarity in the presence of nuisance parameters P. Dawid

Independent inter-departure times in $M / G / I / N$ queues $\quad D . J$. Daley and $D . N$. Shanbhag

Admissibility and minimaxity of some maximum likelihood estimators when the parameter space is restricted to integers $M$. Ghosh

The geometry of generalized inverses W. Kruskal

Addition to the nearest whole unit J.W. Richardson

Coefficients in D-optimal experimental design $R$. Sibson and A. Kenny

Comments and Queries

The consistency of the GEID- (FP-) estimates A. Agren

Issued three times per year (four times in 1975 only). Annual subscription $\mathcal{E 7 . 5 0}$ (U.S. \$18.50) All communications should be addressed to The Secretary, Royal Statistical Society' 21 Bentinck Street, London W1M 6AR, U.K. 


\section{THE PREPARATION OF MANUSCRIPTS}

\section{The attention of authors is particularly directed to the following requests.}

1. Papers should be typed, double-spaced, on one side of white paper (of which A4, $8 \notin$ by $11 \frac{1}{2}$ inches, is a suitable size). The pages must be numbered. Margins of $1 \frac{1}{2}$ inches should be left at the side, top and bottom of each page. The copy sent must be clear.

A cover page should give the title, the author's name and institution, with the address at which mail is to be sent to him.

The title, while brief, must be informative (e.g. A new proof of the prime-number theorem, whereas Some applications of a theorem of G. H. Hardy would be useless).

The first paragraph or two should form a summary of the main theme of the paper, providing an abstract intelligible to mathematicians.

For a typeseript to be aecepted for publieation, it must accord with the standard requirements of publishers, and be presented in a form in which the author's intentions regarding symbols eto. are clear to a printer (who is not a mathematician).

The following notes are intended to help the author in preparing his manuscript. New authors may well enlist the help of a senior colleague, both as to the substance of their work and the details of setting it out correctly and attractively.

2. Notation

Notation should be chosen carefully so that mathematical operations are expressed with all possible neatness, to lighten the task of the compositor and reduce the chance of error.

For instance, $n_{k}(n$ sub $k$ ) is common usage, but avoid if possible using $c$ sub $n$ sub $k$. Fractions are generally best expressed by a solidus. Complicated exponentials like

$$
\exp \left\{z^{2} \sin \theta /\left(1+y^{2}\right)\right\}
$$

should be shown in this and no other way.

In the manuscript, italics, small capitals and capitals are speeified by single, double and triple underlining. Bold-faced type is shown by wavy underlining; wavy will be printed wavy.

It is helpful if displayed equations or statements which will be quoted later in the paper are numbered in order on the right of their line. They can then be referred to by, for example, 'from $(7)$ '.

The author must enable the printer (if necessary by pencilled notes in the margin) to distinguish between similar symbols such as $0,0,0,0,0 ; \mathrm{x}, \mathrm{X} ; \phi, \Phi, \varnothing ; 1,1 ; \epsilon, \epsilon ; \kappa, \mathrm{k}$.

Greek letters can be denoted by $\mathrm{Gk}$ in the margin.

To denote the completion of a proof the sign $\dashv$ may be used.

Footnotes should be avoided.

\section{Diagrams}

It is extremely helpful if diagrams are drawn in indian ink on wbite card, faintly blue or green-lined graph paper, or tracing cloth or paper. Symbols, legends and captions should be given on a transparent overlay. Each text figure must be numbered as Figure 1, Figure 2, ... and its intended position clearly indicated in the manuscript:

\section{Figure 1 here}

The author must peneil his name on all separate sheets of diagrams.

A figure is expensive to reproduce and should be included only when the subject matter demands it, or when it greatly clarifies the exposition.

The Society recognizes that some authors do not have the facilities for producing drawings of a sufficiently high standard to be reproduced directly and it is therefore willing to have such diagrams re-drawn, provided they are reasonably clear.

4. Tables

Tables should be numbered (above the table) and set out on separate sheets. Indicate the position of each in the text as for figures:

Table 3 here

\section{References}

References should be collected at the end of the paper numbered in alphabetical order of the authors' names. A reference to a book should give the title, in italies, and then in Roman type the publisher's name and the place and year of publication:

(4) Jerfaeys, H. The earth, 5th edition, University Press, Cambridge, 1970.

A reference to a paper should give in italies the title of the periodical, the number of the volume and year, and the beginning and end pages of the paper. Titles should be abbreviated as in Mathematical Reviews:

(6) Lrrmewood, J. E. The 'pits effeet' for functions in the unit cirole. J. Analyse Math. 23 (1970), 237-268. 


\section{Mathematical Proceedings of the Cambridge Philosophical Society}

MPCPCO 78 (Pt I) I-I $_{4}$ (1975) $0305-004 \mathrm{I} \quad$ July 1975

CONTENTS

PAGE

RHODEs, C. P. L. A multiplicative property of $R$-sequences and $H_{1}$-sets ～. . . 1

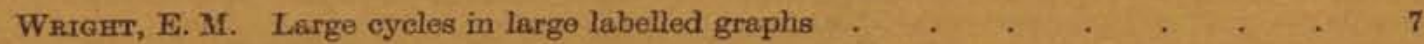

Brown, R., Higenss, P. J. and Morris, S. A. Countable produets and sums of lines and circles: their closed subgroups, quotients and duality properties $\quad$. $\quad . \quad$. $\quad . \quad 19$

Eutrort, P. D. T. A. The law of large numbers for additive arithmetio functions . 33

Brrch, B. J. A look back at Ramanujan's Notebooks . . . . . . . . 73

Grhrnag, F. W. Lower dimensional absolute continuity properties of quasiconformal

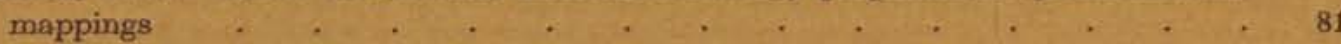

Fremun, D. H. Topological measure spaces: two counter-examples . . . . 95

Frshel, B. Partial isometries which are sums of shifts , . . . . . . 107

BuoNoristiano, S. Handle-theory and engulfing . . . . . . . . . . 111

LAscu, A. T., MUMford, D. and Scotr, D. B. The self-intersection formula and the

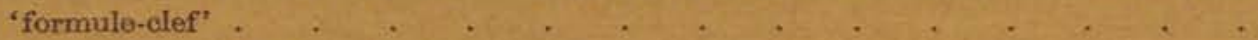

TweenIE, R.L. Sufficient conditions for regularity, recurrence and ergodicity of Markov processes . . . . . . . . . . . .

Merrose, R. B. and Pemberton, M. Periodic solutions of certain nonlinear autonomous wave equations . . . . . . . . . . . . . . 137

ExwIS, J. R. Motion of a classical particle with spin . . . . . . . 145

Chree-Seng, L. Moving foreing effects in a two-phase medium . . . . . 157

(c) The Cambridge Philosophical Society, 1975

CAMBRIDGE UNIVERSITY PRESS

BENTLEY HOUSE, 200 EUSTON ROAD, LONDON NWI $2 \mathrm{DB}$

32 EAST 57 TH STREET, NEW YORK, N.Y. 10022

Price $£ 4.80$ net (U.S.A. and Canada US \$15.50)

1975 subscription price £12 per volume ( $\$ 24$ per annum) net post free

(US $\$ 38.00$ per volume (US $\$ 76.00$ per annum) in U.S.A. and Canada) 